\title{
A case of thalamic abscess treated by endoscopic surgery
}

\author{
Mufique Gajdhar, Y. R. Yadav \\ Department of Neurosurgery Unit, NSCB Medical College, Jabalpur, Madhya Pradesh, India
}

\begin{abstract}
We report about a 7-year-old female child with cyanotic heart disease whose thalamic abscess was successfully treated by endoscope-assisted abscess drainage. Endoscopic aspiration of thalamic abscess appears to be a safe and effective method of treatment for deep-seated abscesses, as direct visualization of the abscess cavity is possible and the completeness of evacuation can be assessed.
\end{abstract}

Key words: Endoscopic surgery; minimal invasive surgery; thalamic abscess.

Thalamic abscesses are relatively rare. The reported incidence varies from 1.3 to $6 \%$ of the brain abscesses. ${ }^{[1],[2]}$ Stereotactic aspiration has been considered to be the treatment of choice. ${ }^{[3],[4]}$ Endoscope-assisted evacuation of thalamic abscess has not been reported. We isolated a report by Jiang et al., where the thalamic abscess was evacuated using stereotactic endoscopy. ${ }^{[5]}$

\section{Case report}

A 7-year-old female child with cyanotic heart disease presented with high-grade fever, headache, vomiting, and altered sensorium with GCS (Glasgow coma scale) score of 13/15 (E3M6V4). The computerized tomography (CT) scan showed an abscess in the right thalamus, which measured approximately $4.7 \mathrm{~cm}$ in its maximum diameter, and projected into the body of the right lateral ventricle [Figure 1]. Hemogram showed mild leucocytosis with increased polymorphs. The patient was started on parenteral antibiotics. The neurological status of the child worsened soon after admission (GCS was 11/15 - E3 M5V3). The child also developed left hemiparesis. Endoscopic method, as advocated by Gaab, was adopted for treatment. Both the lateral ventricles were dilated, making endoscopic evacuation possible without stereotactic guidance. Right frontal burr hole was done $2 \mathrm{~cm}$ anterior to the coronal suture. The abscess was aspirated through the right lateral ventricle, lateral to the choroids plexus. Precaution was taken to avoid contamination in the lateral ventricle as far as possible. A thorough irrigation with antibiotic solution was done and the opening of the abscess cavity was enlarged to ensure free communication between the ventricle and the abscess cavity. An intraventricular drain was placed and continuous irrigation with gentamycin solution was done on a $12 \mathrm{~h}$ basis for 5 days. Repeat CT scan showed complete disappearance of the abscess [Figure 2]. The pus cultured streptococcus viridans and appropriate antibiotics were instituted for 6 weeks. The patient progressively improved in her clinical state. At a follow up after 1 year, the child was found to be asymptomatic.

\section{Discussion}

Thalamic abscesses are uncommon. ${ }^{[1]}$ These are metastatic lesions, underlying sources of infection being congenital heart disease, intrathoracic sepsis, abdominal sepsis, dental caries, otitis media, or sinusitis. ${ }^{[1],[2],[4]}$ Treatment options for thalamic abscess include stereotactic aspiration with or without continuous drainage, ${ }^{[3],[4]}$ free hand aspirations through a burr hole, ${ }^{[4]}$ stereo-endoscopic aspiration, ${ }^{[5]}$ ultrasound guided as-

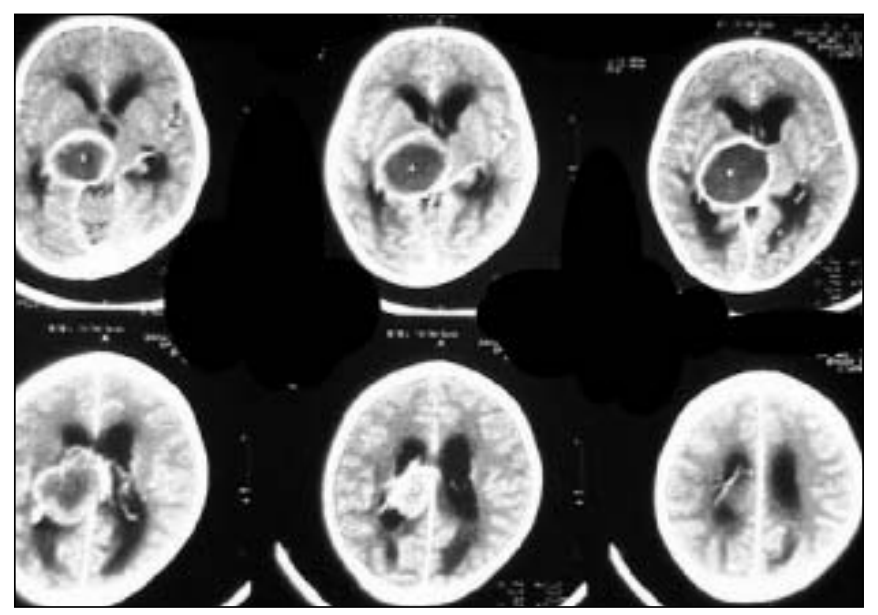

Figure 1: Axial contrast enhanced computerized tomography (CT) scan showing abscess in right thalamus with ventricular dilatation 


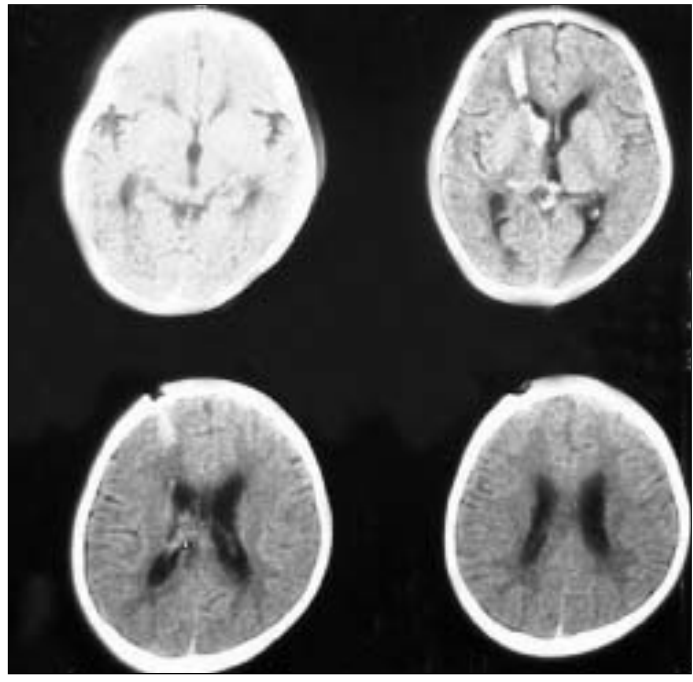

Figure 2: Axial contrast enhanced postoperative computerized tomography (CT) scan showing complete evacuation of thalamic abscess

piration, surgical trans ventricular approach, and medical management.

Stereotactic aspiration remains the treatment of choice as it is minimally invasive, carries minimal morbidity, and can be performed on compromised patients under local anesthesia. ${ }^{[6]}$ Reported incidence of complications that can occur with stereotactic surgery is $7.2 \%$. Symptomatic hemorrhage ranges from 0 to $5.3 \%$ cases with an average of $1.6 \%$ and when it occurs it cannot be controlled. This procedure is followed blindly, and therefore, there are chances of incomplete evacuation and recurrence of abscess. There is also a possibility of spillage of infected material in the ventricle. Ventriculoperitoneal shunt may be required in $50 \%$ of the cases, after stereotactic aspiration or drainage. ${ }^{[4]}$

Evacuation of abscess by a stereotactic endoscope has been reported by Jiang et al. ${ }^{[5]}$ There was no postoperative complication. The endoscopic aspiration with antibiotic irrigation is an alternate method of treatment. There is no report that mentions endoscopic aspiration of thalamic abscess; however, endoscopic treatment of brain abscesses has been described. ${ }^{[7],[8]}$ The advantage of this technique is that the abscess is aspirated under direct visualization, and all loculi can be broken and completeness of aspiration can be directly assessed. Direct visualization in endoscopic surgery also reduces chances of hemorrhage and if it occurs, it can be managed by direct coagulation. There may be difficulty in localization of abscess in smaller lesions, but anatomical landmarks such as choroids plexus and thalamostriate vein along with CT scan morphology, can be used to localize the lesion.

\section{References}

1. Lutz TW, Landott H, Wasner M. Diagnosis and management of abscess in the basal ganglia and thalamus: a survey. J Neurosurg 1984;44:226-32.

2. Bhatia R, Tandon P, Mishra AK. Inflammatory lesion of the basal ganglia and thalamus. Review of twenty one cases. Neurosurgery 1986;19:983-8.

3. Shahzadi S, Lozano AM, Bernstein M, Guha A, Tasker RR. Stereotactic management of bacterial brain abscesses. Can J Neurol Sci.1996;23:34-9.

4. Singh P, Singh A, Thalamic and ganglionic abscesses: a report of two cases: letter to editor. Neurol India 2002;50:225-6.

5. Wan J, Jiang C. Development and clinical application of a brain stereotactic endoscope. Zhonghua Wai Ke Za Zhi 1998;36:536-8.

6. Boviatsis EJ, Kouyialis AT, Stranjalis G, Korfias S, Sakas DE. CT-guided stereotactic aspiration of brain abscesses. Neurosurg Rev. 2003;26:206-9.

7. Hellwig D, Bauer BL, Dauch WA. Endoscopic stereotactic treatment of brain abscesses. Acta Neurochir Suppl 1994;61:102-5.

8. Fritsch M, Manwaring KH. Endoscopic treatment of brain abscesses in children. Minim Invasive Neurosurg 1997:40:103-6.

Accepted on 07-06-2005 